\title{
BRYN MAWR
}

COLLEGE

\section{Bryn Mawr College Open Access Policy}

Approved by the faculty on December 11, 2013

The Faculty of Bryn Mawr College is committed to disseminating the fruits of its research and scholarship as widely as possible. In keeping with that commitment, the Faculty adopts the following policy: Each Faculty member grants to Bryn Mawr College permission to make available her or his scholarly articles and to exercise the copyright in those articles. More specifically, each Faculty member grants to Bryn Mawr College a nonexclusive, irrevocable, worldwide license to exercise any and all rights under copyright relating to each of her or his scholarly articles, in any medium, provided that the articles are not sold for a profit, and to authorize others to do the same. The policy applies to all scholarly articles authored or co-authored while the person is a member of the Faculty of Bryn Mawr College except for any articles completed before the adoption of this policy and any articles for which the Faculty member entered into an incompatible licensing or assignment agreement before the adoption of this policy. The Office of the Provost will waive application of the license for a particular article or delay access for a specified period of time for any reason upon written request from the Faculty member.

Each Faculty member will provide an electronic copy of the author's final version of each article no later than the date of its publication at no charge to the Office of the Provost in an appropriate format as specified by the Office of the Provost. The Office of the Provost will make the article available to the public in an open-access repository. In collaboration with representatives of the Faculty and Information Services, the Office of the Provost will be responsible for interpreting this policy, resolving disputes concerning its interpretation and application, and recommending changes to the Faculty at large from time to time. The Office of the Provost will oversee a yearly review of the policy and present a report to the Faculty. 